\title{
Use of a baseline risk score to identify the risk of serious infectious events in patients with rheumatoid arthritis during certolizumab pegol treatment
}

Jeffrey R. Curtis ${ }^{1 *}$, Kevin Winthrop², Cathy O'Brien ${ }^{3}$, Matladi N. Ndlovu' ${ }^{3}$ Marc de Longueville ${ }^{3}$ and Boulos Haraoui ${ }^{4}$

\begin{abstract}
Background: The risk of serious infectious events (SIES) is increased in patients with rheumatoid arthritis (RA). The aim of this study was to develop an age-adjusted comorbidity index (AACI) to predict, using baseline characteristics, the SIE risk in patients with RA treated with certolizumab pegol (CZP).

Methods: Data of CZP-treated patients with RA were pooled from the RAPID1/RAPID2 randomized controlled trials (RCT CZP) and their open-label extensions (All CZP). Predictors of the first SIE were examined using multivariate Cox models. The AACl was developed by assigning specific weights to patient age and comorbidities on the basis of relative SIE risk. SIE rates were predicted using AACI score and baseline glucocorticoid use, and they were compared with observed rates. The percentage of patients in each SIE risk group achieving low disease activity (LDA)/remission was examined at 1 year of treatment.
\end{abstract}

Results: Among 1224 RCT CZP patients, 40 reported $\geq 1$ SIE (incidence rate [IR] 5.09/100 patient-years [PY]), and 201 of 1506 All CZP patients reported $\geq 1$ SIE (IR 3.66/100 PY). Age $\geq 70$ years, diabetes mellitus, and chronic obstructive pulmonary disease/asthma made the greatest contributions to AACI score. SIE rates predicted using $\mathrm{AACl}$ and glucocorticoid use at baseline showed good agreement with observed SIE rates across low-risk and high-risk groups. At 1 year, more high-risk All CZP patients than low-risk All CZP patients reported SIEs (IR 8.4/100 PY vs. IR 3.4/100 PY). Rates of LDA/remission were similar between groups.

Conclusions: AACl and glucocorticoid use were strong baseline predictors of SIE risk in CZP-treated patients with RA. Predicted SIE risk was not associated with patients' likelihood of clinical response. This SIE risk score may provide a valuable tool for clinicians when considering the risk of infection in individual patients with RA.

Trial registration: ClinicalTrials.gov, NCT00152386 (registered 7 September 2005); NCT00160602 (registered 8 September 2005); NCT00175877 (registered 9 September 2005); and NCT00160641 (registered 8 September 2005).

Keywords: Certolizumab pegol, Safety, Global risk score, Comorbidity, Serious infection

\footnotetext{
*Correspondence: jrcurtis@uabmc.edu

'University of Alabama at Birmingham, Birmingham, AL, USA

Full list of author information is available at the end of the article
} 


\section{Background}

Anti-tumor necrosis factor (anti-TNF) agents are often the first class of biologic drugs prescribed to patients with rheumatoid arthritis (RA), providing an effective therapeutic option that improves clinical, radiographic, and functional outcomes [1]. Owing to their immunomodulatory action, anti-TNFs have been linked to an increased risk of serious infectious events (SIEs), although the strength of this association remains a topic of debate [2-5]. In light of these concerns, biologic registries have been established in several countries to examine the long-term safety of anti-TNFs and to investigate how different patient characteristics affect the risk of serious infection [6-9].

In addition, older age and specific comorbidities that are relatively prevalent in the RA population, such as diabetes mellitus or chronic obstructive pulmonary disease (COPD), have been associated with an increased risk of SIEs during anti-TNF therapy [9-14]. Concomitant treatment with systemic glucocorticoids has also been linked to an increased susceptibility to infection $[6,8,12,15]$. In clinical practice, physicians have to balance the benefits of the different available treatments against their overall risk, as well as the risks associated with the characteristics of each patient. This often involves the complex task of extrapolating data from study populations to the individual patient level. Infection risk scores, which summarize the relative contributions of various patient characteristics in a single composite measure, may help clinicians to anticipate the potential risk of SIEs in individual patients and make better-informed treatment decisions when balancing the expected therapeutic risks and benefits for an individual patient $[11,16,17]$.

Certolizumab pegol (CZP) is a PEGylated, Fc-free antiTNF approved for the treatment of adult patients with moderate to severe active RA [18-21]. Using baseline patient characteristics, we developed and tested an ageadjusted comorbidity index (AACI) to predict the risk of SIEs in patients with RA at the start of CZP treatment. In addition, we investigated if the predicted SIE risk was associated with the likelihood of clinical response at 1 year of CZP treatment.

\section{Methods}

\section{Patient population and study design}

To develop an AACI predictive of SIE risk in patients with RA initiating CZP treatment, we pooled baseline data from anti-TNF naive patients with RA who participated in the Rheumatoid Arthritis PreventIon of structural Damage 1 (RAPID1) and RAPID2 randomized controlled trials (RCTs; ClinicalTrials.gov, NCT00152386 and NCT00160602, respectively) and their respective openlabel extensions (OLEs; NCT00175877 and NCT00160641, respectively). These pivotal registration studies have been described in detail elsewhere [20-23], and their study design is summarized in Fig. 1.

Oral glucocorticoids ( $\leq 10 \mathrm{mg} /$ day prednisone equivalent) were permitted, provided that doses remained stable within 28 days of baseline and throughout the RCTs; doses were allowed to change during the OLEs. Additional details concerning patients' eligibility criteria and permitted medications are reported elsewhere [20, 21].

\section{Definition of serious infectious events and patient groups analyzed}

SIEs were classified according to the Medical Dictionary for Regulatory Activities (MedDRA) version 9.0. The definition of SIE encompassed the regulatory definition of serious adverse event (SAE) of infection [24] plus any medical events deemed important by the investigator, regardless of infection severity. All suspected SIEs were subsequently expert-reviewed by an external independent safety steering committee that classified SIEs by the regulatory definition of SAE of infection, with an additional criterion of the need for intravenous antibiotics [25].

For each patient, analyses included the first SIE that occurred after the first dose of CZP and up to 84 days (six times the half-life of CZP) after the last study dose or patient withdrawal; any subsequent SIEs were not included. Two overlapping patient groups were analyzed: (1) patients randomized to CZP in the RCTs (RCT CZP; only SIEs occurring during the RCTs were included) and (2) all patients treated with CZP during the RCTs and/or OLEs, including RCT placebo completers switched to CZP at the start of OLE as well as patients withdrawn from the RCTs at week 16 who reconsented to enter the OLE (All CZP; SIEs occurring during the RCTs or OLEs were included).

\section{Derivation of age-adjusted comorbidity index}

An AACI was developed to predict the influence of baseline age and medically treated comorbidities on the risk of SIEs during CZP treatment. The AACI was conceptually similar to the Charlson comorbidity index [26], but it was designed to better reflect the age and specific comorbidity burden of an RA patient population similar to participants in the RAPID1/RAPID2 trials. Age at baseline $(<50, \geq 50$ to $<60, \geq 60$ to $<70$, and $\geq 70$ years $)$ and the most frequent medically treated comorbidities (diabetes mellitus, COPD/asthma, cardiac disorder [including coronary artery disease and heart failure], hypertension, hyperlipidemia, thyroid disorder, osteoporosis, and depression) were considered for inclusion in the AACI. Medically treated comorbidities were identified on the basis of patients' medical histories or medications at baseline.

Using the RCT CZP data, the weight attributed to each baseline covariate in the AACI was derived by including all candidate covariates (described below) in a 


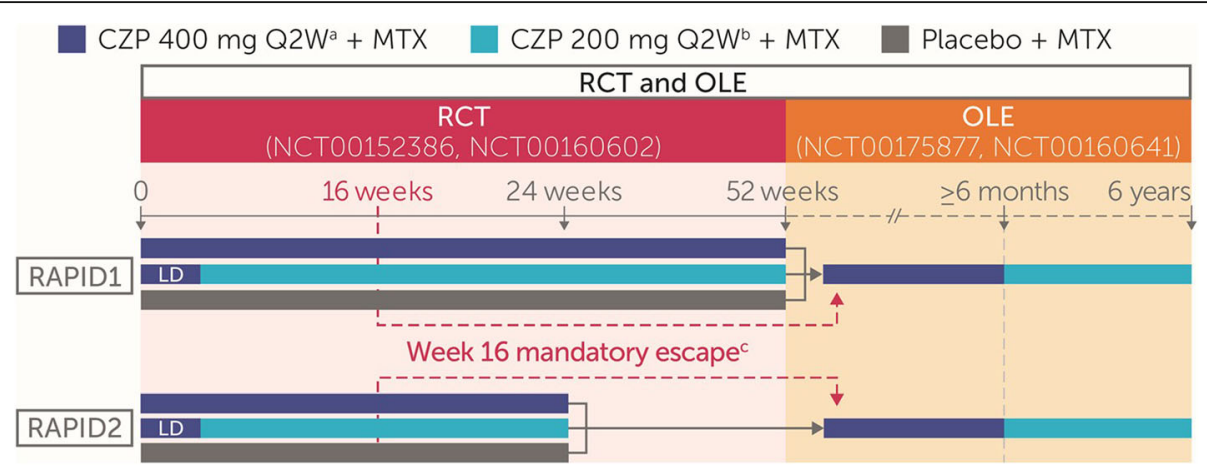

Fig. 1 Rheumatoid Arthritis Preventlon of structural Damage 1 (RAPID1) and RAPID2 study design. Patients were randomized 2:2:1 to CZP $400 \mathrm{mg}$ Q2W, CZP $200 \mathrm{mg}$ Q2W, or placebo Q2W, respectively, in combination with MTX. The loading dose (LD) was CZP $400 \mathrm{mg}$

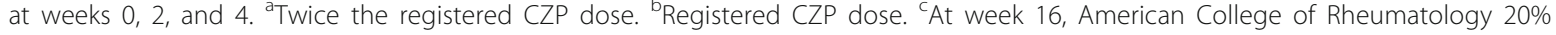
improvement nonresponders at weeks 12 and 14 were withdrawn from the RCTs; these patients, as well as those who completed the RCTs, were allowed to enter the OLEs, receiving CZP $400 \mathrm{mg}$ Q2W + MTX for $\geq 6$ months before being switched to CZP $200 \mathrm{mg}$ Q2W + MTX. CZP Certolizumab pegol, Q2W Every 2 weeks, Q4W Every 4 weeks, MTX Methotrexate, RCT Randomized controlled trial, OLE

Open-label extension

multivariate Cox proportional hazards model to estimate the relative risk of SIEs associated with each factor. The same analysis was repeated using the All CZP data. The two HRs obtained for each covariate in the RCT CZP and All CZP analyses were averaged to assign the corresponding weight $(\mathrm{HR}<1.2$, weight $=0 ; \mathrm{HR} \geq 1.2$ and $<1.5$, weight $=1 ; \mathrm{HR} \geq 1.5$ and $<2.5$, weight $=2$; $\mathrm{HR} \geq 2.5$ and $<3.5$, weight $=3$ ). An individual patient's AACI score corresponded to the sum of the weights associated with their age and specific comorbidities (Table 1 and Additional file 1: Table S1).

\section{Evaluation of risk of SIEs}

Observed incidence rates (IRs) of SIEs per 100 patientyears (PY) together with 95\% CIs were calculated. Time at risk was measured from initiation of CZP up to the occurrence of the first SIE, or the total time at risk was measured for patients without SIEs (up to 84 days after the last study

Table 1 Relative serious infectious event risk associated with baseline age categories and medically treated comorbidities included in age-adjusted comorbidity index

\begin{tabular}{|c|c|c|c|c|}
\hline \multirow{2}{*}{ Category } & & \multicolumn{2}{|l|}{ HR $(95 \%$ Cl) } & \multirow{2}{*}{$\begin{array}{l}\text { Weight } \\
\text { in the } \\
\mathrm{AACl}^{\mathrm{a}}\end{array}$} \\
\hline & & $\overline{\mathrm{RCT}} \mathrm{CZP}(n=1224)$ & All CZP $(n=1506)$ & \\
\hline \multirow[t]{4}{*}{ Age, years } & $<50$ & Reference & Reference & 0 \\
\hline & $\geq 50$ to $<60$ & $1.29(0.58-2.87)$ & $1.39(0.99-1.96)$ & 1 \\
\hline & $\geq 60$ to $<70$ & $1.14(0.44-2.94)$ & $1.40(0.92-2.12)$ & 1 \\
\hline & $\geq 70$ & $2.18(0.70-6.84)$ & $2.93(1.69-5.09)$ & 3 \\
\hline Diabetes mellitus & & $1.98(0.59-6.58)$ & $1.61(0.90-2.89)$ & 2 \\
\hline COPD/asthma & & $2.67(0.77-9.27)$ & $1.29(0.56-2.97)$ & 2 \\
\hline Cardiac disorder & & $\mathrm{N} / \mathrm{C}$ & $1.33(0.52-3.43)$ & 0 \\
\hline Hypertension & & $1.34(0.69-2.63)$ & $0.96(0.70-1.32)$ & 0 \\
\hline Hyperlipidemia & & $2.39(0.82-6.93)$ & $1.47(0.81-2.67)$ & 2 \\
\hline Thyroid disorder & & $\mathrm{N} / \mathrm{C}$ & $0.87(0.42-1.78)$ & 0 \\
\hline Osteoporosis & & $2.30(0.98-5.39)$ & $1.16(0.73-1.86)$ & 2 \\
\hline Depression & & $1.00(0.22-4.51)$ & $1.46(0.75-2.83)$ & 1 \\
\hline
\end{tabular}

Abbreviations: SIE Serious infectious event, $A A C I$ Age-adjusted comorbidity index, CZP Certolizumab pegol, COPD Chronic obstructive pulmonary disease, N/C Not calculable (no patients with the indicated comorbidity experienced an SIE during the randomized controlled trials), $R C T$ CZP Patients randomized to CZP in the RAPID1/RAPID2 randomized controlled trials, All CZP All patients treated with CZP during the RAPID1/RAPID2 randomized controlled trials and/or open label extensions

HRs were derived from a Cox proportional hazards model fitted with the indicated age categories and medically treated comorbidities; no other baseline covariates were included

${ }^{a}$ Weight of each category in the AACl was based on the average HR between the RCT CZP and All CZP populations (HR $\geq 1.2$ and $<1.5$, weight $=1 ; \mathrm{HR} \geq 1.5$ and $<2.5$, weight $=2 ; \mathrm{HR} \geq 2.5$ and $<3.5$, weight $=3$ ) 
dose or patient withdrawal). Placebo-treated patients did not contribute any data to the analysis until they entered the OLE and began treatment with CZP.

To identify all clinically relevant predictors of SIEs, a stepwise Cox proportional hazards model was fitted with AACI categories $(0,1, \geq 2)$ and additional baseline covariates: body mass index (BMI; $<20,20-30$, or $>30 \mathrm{~kg} / \mathrm{m}^{2}$ ), 28-joint Disease Activity Score with C-reactive protein (DAS28[CRP]), disease duration ( $<2$ or $\geq 2$ years), Health Assessment Questionnaire Disability Index (HAQ-DI), joint erosion score (computed as $\log [$ erosion score] and included as a proxy for disease severity), methotrexate dose ( $\leq 15$ or $>15 \mathrm{mg} /$ week), and systemic glucocorticoid use (yes or no). Covariates were retained in the Cox model if there was an indication of a link with the occurrence of the first SIE $(p \leq 0.25)$; covariates with $p \leq 0.05$ were identified as risk factors. All $p$ values were nominal only. RCT CZP and All CZP patients were then categorized into six different risk groups based on AACI categories $(0,1, \geq 2)$ and systemic glucocorticoid use (yes or no).

The Kaplan-Meier estimator was used to analyze the time to first SIE. Predicted SIE rates were based upon each patient's covariate distribution and were obtained at regular time intervals up to the date of the last recorded SIE in RCT CZP (day 301) and up to 2 years in the All CZP group. The ability of the model to discriminate between risk groups was evaluated by calculating the c-index [27]. As a sensitivity analysis, the Rheumatoid Arthritis Observation of Biologic Therapy (the German biologics register) (RABBIT) Risk Score [17] was tested in the All CZP group (see Additional file 1: Methods).

\section{Clinical outcomes}

For each of the six SIE risk groups in All CZP, the percentage of patients with low disease activity (LDA; DAS28[CRP] $\leq 2.7$, Clinical Disease Activity Index $[C D A I] \leq 10$, and Simplified Disease Activity Index $[\mathrm{SDAI}] \leq 11)$ and in remission (DAS28[CRP] $<2.3$, CDAI $\leq 2.8$, and SDAI $\leq 3.3$ ) was evaluated at 1 year of CZP exposure. Missing data were imputed using nonresponder imputation.

\section{Results}

\section{Patient population and overall incidence of SIEs}

The anti-TNF naive RA population pooled from RAPID1 and RAPID2 comprised 1224 patients in the RCT CZP group, with a total CZP exposure of 798.5 PY and median exposure per patient of $0.5 \mathrm{PY}$. The All CZP group comprised 1506 patients (including 282 of 311 RCT placebo patients and 1074 of 1224 RCT CZP patients who reconsented to enter the OLEs) with a total CZP exposure of 5778.6 PY and median exposure per patient of 4.8 PY. Demographic characteristics, baseline disease activity, and the prevalence of medically treated comorbidities were similar between the two patient groups (Table 2). Over $50 \%$ of patients in each group used systemic glucocorticoids at baseline.

During the RCTs, 40 of 1224 RCT CZP patients reported $\geq 1$ SIE (IR 5.09/100 PY [3.64-6.93]). Over the combined RCT and OLE periods, 201 of 1506 All CZP patients reported $\geq 1$ SIE (IR 3.66/100 PY [3.17-4.21]). Pneumonia and cellulitis were the most common SIEs (39 and 16 events, respectively, in All CZP). Overall, 15 RCT CZP patients and 69 All CZP patients were withdrawn from the studies because of SIEs.

\section{Contribution of age and comorbidities selected for inclusion in age-adjusted comorbidity index}

Age $\geq 70$ years was strongly associated with the risk of SIEs, contributing 3 points to the AACI score. Diabetes mellitus and COPD/asthma were also important predictors of SIEs and were assigned a weight of 2 points each (Table 1). Other comorbidities contributing to AACI score were hyperlipidemia, osteoporosis, and depression.

Among all patients, the distribution of AACI scores ranged between 0 and 8 , and the observed percentage of patients with SIEs generally increased with AACI score (Additional file 1: Table S1). Most patients (80\% in both groups) had AACI scores $\leq 1$; only one patient in the All CZP group had an AACI score of 8 . Owing to the limited number of patients with high AACI scores, those with AACI scores $\geq 2$ were pooled in subsequent analyses.

\section{Identification of baseline risk factors for first SIE}

AACI scores $\geq 2$ and baseline systemic glucocorticoid use were identified by the Cox model as the main risk factors for the first SIE (Fig. 2). In both RCT CZP and All CZP, an AACI $\geq 2$ was associated with an increase in the risk of SIEs compared with AACI of 0 (RCT CZP HR 2.86 [1.23-6.62]; All CZP HR 2.59 [1.79-3.76]), and an AACI of 1 was also associated with increased SIE risk in All CZP (HR 1.42 [1.01-2.01]). Baseline systemic glucocorticoid use was also associated with an increased risk of SIEs in RCT CZP patients (HR 2.33 [1.10-4.95]). The association was weaker in the All CZP group (HR 1.26 [0.95-1.68]). In a sensitivity analysis examining different baseline glucocorticoid doses, the risk of SIEs was similar between glucocorticoid doses $>0-5 \mathrm{mg} /$ day and $>5 \mathrm{mg} /$ day (data not shown).

Baseline DAS28(CRP) and erosion scores did not contribute to SIE risk in the All CZP group, but they showed a modest association in the RCT CZP group. No other covariates tested (BMI, disease duration, HAQ-DI, and MTX dose) showed a detectable association with the risk of SIEs $(p>0.25)$. Replacing DAS28(CRP) in the Cox model with alternative measures of disease activity (CRP, DAS28[erythrocyte sedimentation rate], CDAI, and SDAI) 
Table 2 Baseline patient characteristics

\begin{tabular}{|c|c|c|c|}
\hline & & $\mathrm{RCT}$ CZP $(n=1224)$ & All CZP $(n=1506)$ \\
\hline Female sex, $n(\%)$ & & $1008(82.4)$ & $1245(82.7)$ \\
\hline \multirow[t]{4}{*}{ Age, years, $n(\%)$} & $<50$ & $470(38.4)$ & $580(38.5)$ \\
\hline & $\geq 50$ to $<60$ & $440(35.9)$ & $546(36.3)$ \\
\hline & $\geq 60$ to $<70$ & $247(20.2)$ & $300(19.9)$ \\
\hline & $\geq 70$ & $67(5.5)$ & $80(5.3)$ \\
\hline \multirow[t]{2}{*}{ Disease duration (years), $n$ (\%) } & $<2$ & $261(21.3)$ & $323(21.4)$ \\
\hline & $\geq 2$ & $963(78.7)$ & $1183(78.6)$ \\
\hline DAS28(CRP), mean (SD) & & $6.20(0.83)$ & $6.20(0.85)$ \\
\hline HAQ-DI, mean (SD) & & $1.65(0.60)$ & $1.65(0.60)$ \\
\hline CRP, mg/L, median (IQR) & & $15.0(6.0-32.0)$ & $15.0(6.0-32.0)$ \\
\hline Rheumatoid factor, IU/ml, median (IQR) & & $68.3(18.9-201.1)$ & $70.0(19.4-200.0)$ \\
\hline mTSS, median (IQR) & & $20.0(5.5-54.7)$ & $20.0(6.0-55.0)$ \\
\hline \multirow[t]{3}{*}{$\mathrm{BMI}, \mathrm{kg} / \mathrm{m}^{2}, n(\%)$} & $<20$ & $83(6.8)$ & $104(6.9)$ \\
\hline & $20-30$ & $833(68.1)$ & $1024(68.0)$ \\
\hline & $>30$ & $308(25.2)$ & $378(25.1)$ \\
\hline \multirow[t]{3}{*}{ Glucocorticoid dose, mg/day, n (\%) } & 0 & $518(42.3)$ & $637(42.3)$ \\
\hline & $>0-5$ & $332(27.1)$ & $402(26.7)$ \\
\hline & $>5$ & $374(30.6)$ & $467(31.0)$ \\
\hline \multirow[t]{2}{*}{ Systemic MTX dose, mg/week, $n(\%)$} & $\leq 15$ & $1018(83.2)$ & $1261(83.7)$ \\
\hline & $>15$ & $206(16.8)$ & $245(16.3)$ \\
\hline \multicolumn{4}{|l|}{ Medically treated comorbidities, $n(\%)^{a}$} \\
\hline Diabetes mellitus & & $47(3.8)$ & $53(3.5)$ \\
\hline COPD/asthma & & $34(2.8)$ & $38(2.5)$ \\
\hline Cardiac disorder ${ }^{\mathrm{b}}$ & & $15(1.2)$ & $17(1.1)$ \\
\hline Hypertension $^{c}$ & & $387(31.6)$ & $465(30.9)$ \\
\hline Hyperlipidemia & & $55(4.5)$ & $66(4.4)$ \\
\hline Thyroid disorder & & $55(4.5)$ & $63(4.2)$ \\
\hline Osteoporosis & & $92(7.5)$ & $113(7.5)$ \\
\hline Depression & & $37(3.0)$ & $45(3.0)$ \\
\hline
\end{tabular}

Abbreviations: CZP Certolizumab pegol, RCT Randomized controlled trial, DAS28(CRP) 28-joint Disease Activity Score with C-reactive protein, CRP C-reactive protein, $H A Q-D I$ Health Assessment Questionnaire Disability Index, mTSS Modified total Sharp score, MTX Methotrexate, BMI Body mass index, COPD Chronic obstructive pulmonary disease, $R C T$ CZP Patients randomized to CZP in the RAPID1/RAPID2 randomized controlled trials, All CZP All patients treated with CZP during the RAPID1/RAPID2 randomized controlled trials and/or open label extensions

${ }^{a}$ Medically treated comorbidities were identified on the basis of patients' medical histories and medications at baseline

${ }^{\mathrm{b}}$ Cardiac disorder included coronary artery disease and heart failure

${ }^{\mathrm{C}}$ Hypertension included patients with history of cerebrovascular disorder ( $n=5$ in RCT CZP and $n=5$ in All CZP) and transient ischemic attack ( $n=4$ in RCT CZP and $n=5$ in All CZP)

produced results similar to those of the main analyses (data not shown).

\section{Use of baseline age-adjusted comorbidity index and systemic glucocorticoids to predict rate of SIES}

In the RCT CZP group, the predicted risk of SIEs was lowest for patients with an AACI of 0 and no systemic glucocorticoid use at baseline, and highest in patients with an AACI $\geq 2$ who used systemic glucocorticoids (Fig. 3a). Within the same AACI category, the percentage of patients reporting a first SIE was approximately twofold higher if patients also used systemic glucocorticoids at baseline. The c-index $(0.66$ [0.33-0.93]) indicated fair discrimination between patient groups.

In the All CZP group, the predicted risk of SIEs was lowest for the patient groups defined by AACI of 0 and highest for patients with AACI $\geq 2$, regardless of systemic glucocorticoid use (Fig. 3b). The c-index (0.85 [0.73-0.93]) suggested excellent discrimination between patient groups.

Across the different risk groups, observed SIE rates generally matched model predictions, demonstrating that the prediction model was well-calibrated (Table 3). 


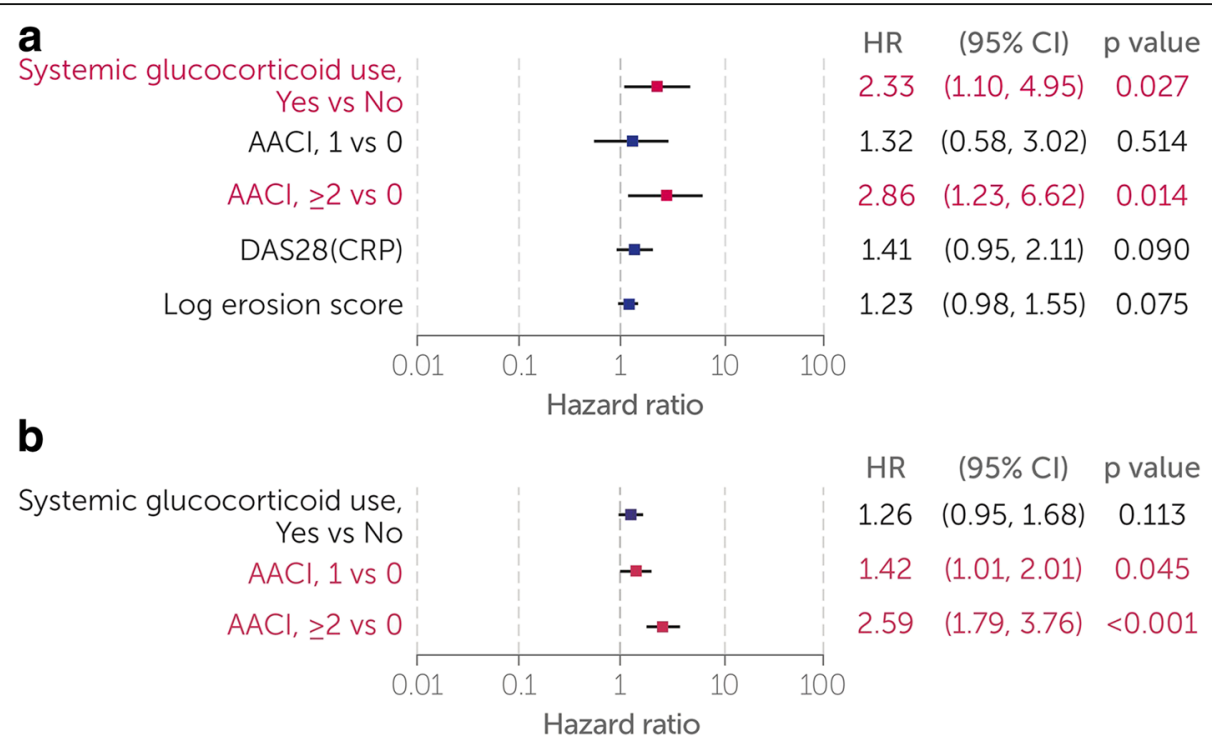

Fig. 2 Multivariate analysis of baseline predictors to the first SIE. a RCT CZP group. b All CZP group. A Cox proportional hazards model fitted with AACl categories was used to identify baseline covariates linked to SIE risk $(p \leq 0.25)$. Baseline covariates identified as risk factors $(p \leq 0.05)$ are highlighted in red. No other baseline covariates examined (BMI, disease duration, HAQ-DI, and MTX dose) were considered relevant to the outcome $(p>0.25)$. SIE Serious infectious event, RCT Randomized controlled trial, CZP Certolizumab pegol, AACI Age-adjusted comorbidity index, DAS28(CRP) 28-joint Disease Activity Score with C-reactive protein, BMI Body mass index, HAQ-DI Health Assessment Questionnaire Disability Index, MTX Methotrexate, RCT CZP Patients randomized to CZP in the RAPID1/RAPID2 randomized controlled trials, All CZP All patients treated with CZP during the RAPID1/RAPID2 randomized controlled trials and/or open label extensions

Consistent with model predictions, the observed IRs of SIEs were higher for patients with AACI $\geq 2$ and baseline systemic glucocorticoid use, and lower for patients with AACI of 0 and no baseline systemic glucocorticoid use (Table 4).

As a sensitivity analysis, the RABBIT Risk Score was tested in the All CZP group. On the basis of expected SIE risk, patients were assigned only to the first three deciles of RABBIT Risk Scores published by Zink et al. [17]. Predicted SIE rates were generally comparable to observed rates, with some underestimation of SIE rates in patients with lower RABBIT Risk Scores (Additional file 1: Figure S1).

\section{Observed rates of LDA and remission in different SIE risk groups}

We investigated if the predicted SIE risk influenced the achievement of LDA and remission at 1 year of CZP treatment according to DAS28(CRP), CDAI, and SDAI criteria (Fig. 4). In All CZP, the percentage of patients achieving LDA was comparable across the different SIE risk groups, albeit with a numerically higher proportion in the low-risk group (AACI of 0 and no baseline systemic glucocorticoid use). Likewise, the rates of remission did not vary considerably between SIE risk groups.

\section{Discussion}

Patients with RA are reported to have an increased susceptibility to infection compared with patients without RA [28]. Although the strength of association between anti-TNFs and the risk of infection remains a topic of debate [2-5], the evidence published so far underscores the need for clinicians to carefully balance the clinical benefits and potential harms of initiating anti-TNF therapy in individual patients.

Using data pooled from the pivotal RAPID1 and RAPID2 RCTs and OLEs [20-23], we derived and tested a new AACI to predict the risk of SIEs during CZP treatment, based on the baseline characteristics of anti-TNF naive patients with moderate to severe active RA and inadequate response to prior diseasemodifying antirheumatic drugs. AACI $\geq 2$ and systemic glucocorticoid use were identified as the main baseline risk factors for the occurrence of SIEs. With up to 2 years of CZP exposure, predicted SIE rates matched the observed rates, suggesting that the model was well-calibrated for the outcome. Discrimination between different risk groups in the model was fair (RCT CZP) to excellent (All CZP).

As expected, the IRs of SIEs in groups defined as being at high risk were higher than reported previously in a pooled safety analysis of patients with RA in CZP clinical trials (IR 3.7/100 PY [3.3-4.1] [25]). Consistent with previous CZP safety data [25] and registry data $[10,13]$, the highest incidence of SIEs was seen during the first 6 months of anti-TNF treatment and decreased thereafter. 

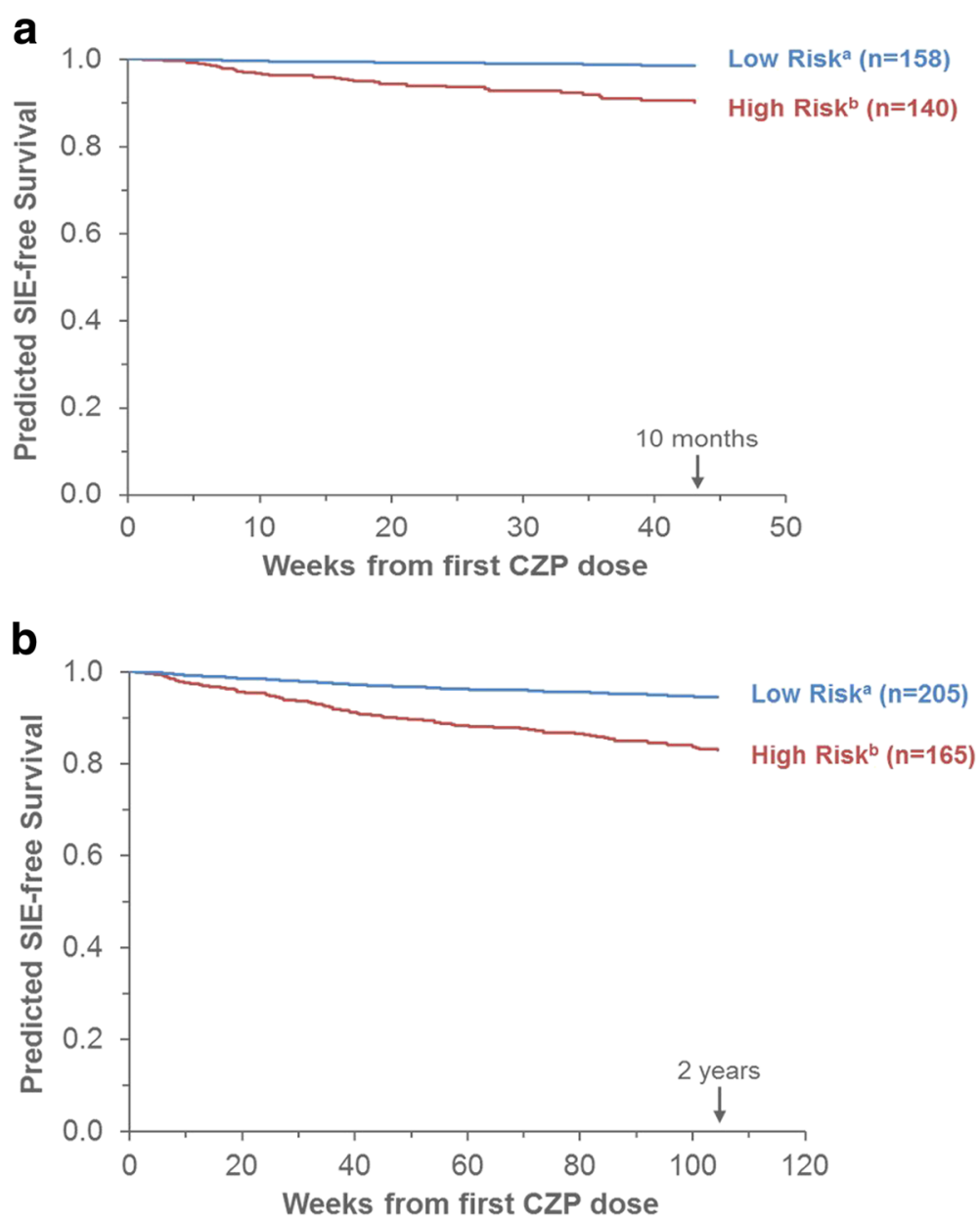

Fig. 3 Predicted time to first SIE, by baseline systemic glucocorticoid use and AACI. a RCT CZP. b All CZP. Predicted SIE-free survival curves correspond to Kaplan-Meier estimates for the indicated risk groups, based on the covariates selected by the Cox models. The c-indexes were 0.66 (95\% Cl 0.33-0.93) for the RCT CZP model and 0.85 (95\% Cl 0.73-0.93) for the All CZP model. ' Low risk at baseline: AACl of 0, without systemic glucocorticoid use. ${ }^{b}$ High risk at baseline: $\mathrm{AACl} \geq 2$, with systemic glucocorticoid use. SIE Serious infectious event, CZP Certolizumab pegol, AACI Age-adjusted comorbidity index, RCT CZP Patients randomized to CZP in the RAPID1/RAPID2 randomized controlled trials, All CZP All patients treated with CZP during the RAPID1/RAPID2 randomized controlled trials and/or open label extensions, Cl Confidence interval

In All CZP, the rates of LDA and remission observed at 1 year were similar across all six risk groups, suggesting that the baseline risk of SIEs was not associated with the likelihood of response to CZP treatment. Indeed, poor prognostic factors for RA progression, such as longer disease duration, greater disability, and erosive disease $[29,30]$, were not associated with SIE risk when tested in the Cox model.

In line with previous studies [9-14], older age ( $\geq 70$ years), diabetes mellitus, and COPD/asthma were strongly associated with SIE risk in this RA population, making an important contribution to patients' AACI scores. Consistent with our results, older age and comorbidities also featured in other SIE risk scores in RA, developed using data from biologics registries and administrative databases [11, 16, 17]. In addition, we found that hyperlipidemia, osteoporosis, and depression were associated with an increased risk of SIEs in the RAPID1/RAPID2 patient population. Depression and the use of certain antidepressant medications have previously been associated with an increased risk of some infections [31]. However, it is uncertain whether some of these associations might have a true biologic basis, be driven by the effects of medications used to treat the condition, be a proxy for a shared risk factor (e.g., frailty), or reflect other confounding factors.

The increased risk of infection associated with systemic glucocorticoids is widely recognized $[6,8,12,15]$. Our data revealed a dose-independent association between baseline systemic glucocorticoid use and SIEs in the RCT CZP group, where doses were required to remain stable throughout the RCTs [20, 21]. By contrast, systemic glucocorticoid doses were allowed to change during the OLEs, which may have minimized our ability to detect a stronger association with SIE 


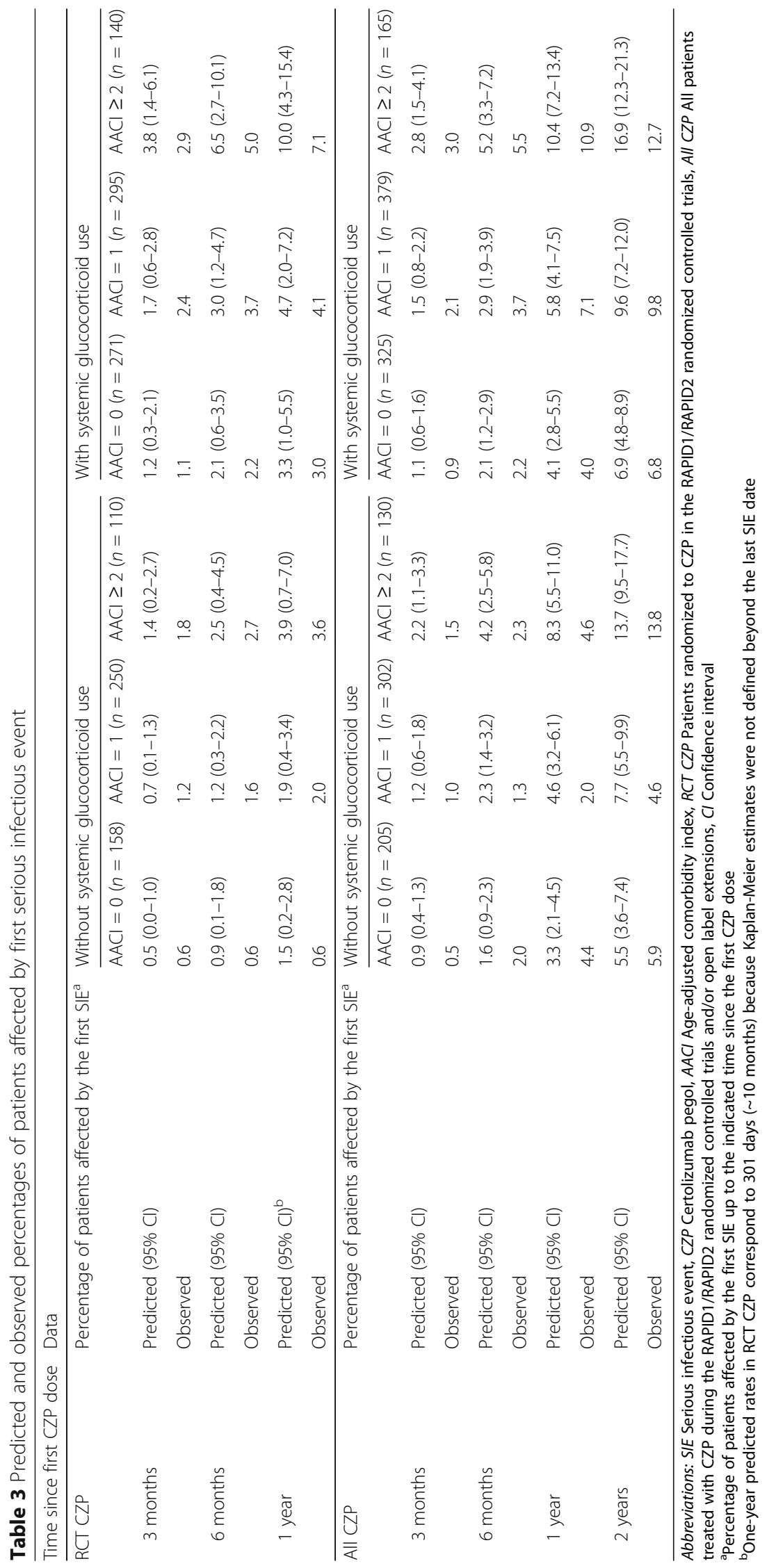


Table 4 Observed incidence of serious infectious events in patients categorized by age-adjusted comorbidity index and baseline systemic glucocorticoid use

\begin{tabular}{|c|c|c|c|c|c|c|}
\hline \multirow{3}{*}{$\begin{array}{l}\text { Time since } \\
\text { first CZP } \\
\text { dose }\end{array}$} & \multicolumn{6}{|c|}{ IR/100 PY $(95 \% C l)^{a}$} \\
\hline & \multicolumn{3}{|c|}{ Without systemic glucocorticoid use } & \multicolumn{3}{|c|}{ With systemic glucocorticoid use } \\
\hline & $\mathrm{AACl}=0$ & $\mathrm{AACl}=1$ & $\mathrm{AACl} \geq 2$ & $\mathrm{AACl}=0$ & $\mathrm{AACl}=1$ & $\mathrm{AACl} \geq 2$ \\
\hline \multicolumn{7}{|l|}{ RCT CZP } \\
\hline Patients, $n$ & 158 & 250 & 110 & 271 & 295 & 140 \\
\hline 3 months & $2.6(0.1-14.5)$ & $4.9(1.0-14.3)$ & $7.8(1.0-28.2)$ & $4.5(0.9-13.3)$ & $9.8(3.9-20.2)$ & $11.9(3.2-30.5)$ \\
\hline 6 months & $1.4(0.0-8.0)$ & $3.7(1.0-9.6)$ & $6.8(1.4-19.9)$ & $5.1(1.9-11.1)$ & $8.6(4.3-15.4)$ & $12.1(4.9-25.0)$ \\
\hline 1 year & $1.0(0.0-5.3)$ & $3.2(1.0-7.4)$ & $6.2(1.7-15.9)$ & $4.6(2.0-9.1)$ & $6.2(3.2-10.9)$ & $12.1(5.8-22.2)$ \\
\hline \multicolumn{7}{|l|}{ All CZP } \\
\hline Patients, $n$ & 205 & 302 & 130 & 325 & 379 & 165 \\
\hline 3 months & $2.0(0.1-11.1)$ & $4.1(0.8-11.9)$ & $6.6(0.8-23.7)$ & $3.8(0.8-11.1)$ & $8.7(3.8-17.1)$ & $12.6(4.1-29.4)$ \\
\hline 6 months & $4.1(1.1-10.5)$ & $2.8(0.8-7.1)$ & $5.1(1.0-14.8)$ & $4.5(1.8-9.3)$ & $7.8(4.3-13.1)$ & $11.7(5.4-22.2)$ \\
\hline 1 year & $4.8(2.2-9.1)$ & $2.2(0.8-4.7)$ & $5.3(2.0-11.6)$ & $4.3(2.3-7.4)$ & $8.0(5.3-11.7)$ & $12.6(7.5-20.0)$ \\
\hline 2 years & $3.4(1.8-6.0)$ & $2.7(1.5-4.5)$ & $8.9(5.3-14.0)$ & $3.9(2.5-5.9)$ & $6.0(4.3-8.3)$ & $8.4(5.2-12.8)$ \\
\hline
\end{tabular}

Abbreviations: SIE Serious infectious event, CZP Certolizumab pegol, IR Incidence rate, PY Patient-years, AACI Age-adjusted comorbidity index, RCT CZP Patients randomized to CZP in the RAPID1/RAPID2 randomized controlled trials, All CZP All patients treated with CZP during the RAPID1/RAPID2 randomized controlled trials and/or open label extensions, $\mathrm{Cl}$ Confidence interval

${ }^{a}$ IR of SIEs observed during the indicated time interval since the first day on CZP

risk in the All CZP group. Furthermore, we cannot discount the possibility that doses taken over the previous 2-3 years may have had a cumulative impact on the risk of SIEs [32]. Despite such limitations, our results suggest potential benefits of avoiding concomitant systemic glucocorticoid use in CZP-treated patients with RA as early as possible, as recommended by the 2015 American College of Rheumatology RA guidelines [33].

High disease activity has been associated with an increased risk of infection [34, 35]. However, in our model, baseline disease activity showed only a modest association with the risk of SIEs. One likely explanation is the fact that all patients enrolled in the RAPID1/RAPID2 RCTs had moderate to high disease activity at baseline. The relatively narrow range of baseline DAS28(CRP) scores in this RA population may have concealed a stronger association with SIE risk.

Several studies have reported that the relative risk of infection decreases over time in patients with RA treated with anti-TNF [13, 25, 36-38]. This has been attributed not only to the depletion of susceptible patients from the study cohorts but also to an improvement in the clinical status of patients, either as a direct result of reduced disease activity or owing to a decline in the use of concomitant glucocorticoids [8]. The model that we developed tested only the effect of baseline patient characteristics. Although AACI, which is a static patient characteristic, was the strongest predictor of SIEs, clinical parameters such as disease activity and physical function (HAQ-DI) may have changed considerably over time as a result of CZP treatment, resulting in weaker prediction of SIE rates for CZP exposures $>2$ years.

A limitation of the method used to derive the AACI is the fact that the HRs for each age category and individual comorbidity were averaged across the RCT CZP and All CZP groups in order to assign the respective weighting. Our intention with the AACI was to provide an indication of SIE risk not only in the first year of treatment but also over a longer period of time. Had we used just the RCT period to derive the weightings for each age category and comorbidity, we would have potentially overestimated the risk of SIEs during the OLE. For this reason, the averaging of HRs seemed to be a reasonable approach, albeit at the expense of some predictive accuracy.

Prior epidemiologic work has suggested that although some risk estimation models can be generalized, model performance is optimized when population-specific weights can be derived [11]. As such, the weighting for each risk factor in one population may not be generalizable to a different RA population. For instance, the RABBIT Risk Score performed well in the All CZP group, but SIE rates were underestimated at the lower end of the risk spectrum. One possible explanation is the fact that the RABBIT Risk Score may be better calibrated for real-world patient populations with a higher propensity for SIEs [17]. For example, chronic renal disease and chronic lung disease, which contribute to the RABBIT Risk Score, were less prevalent or absent in our study population. Furthermore, our assumption that there were zero patients with a history of prior SIEs 


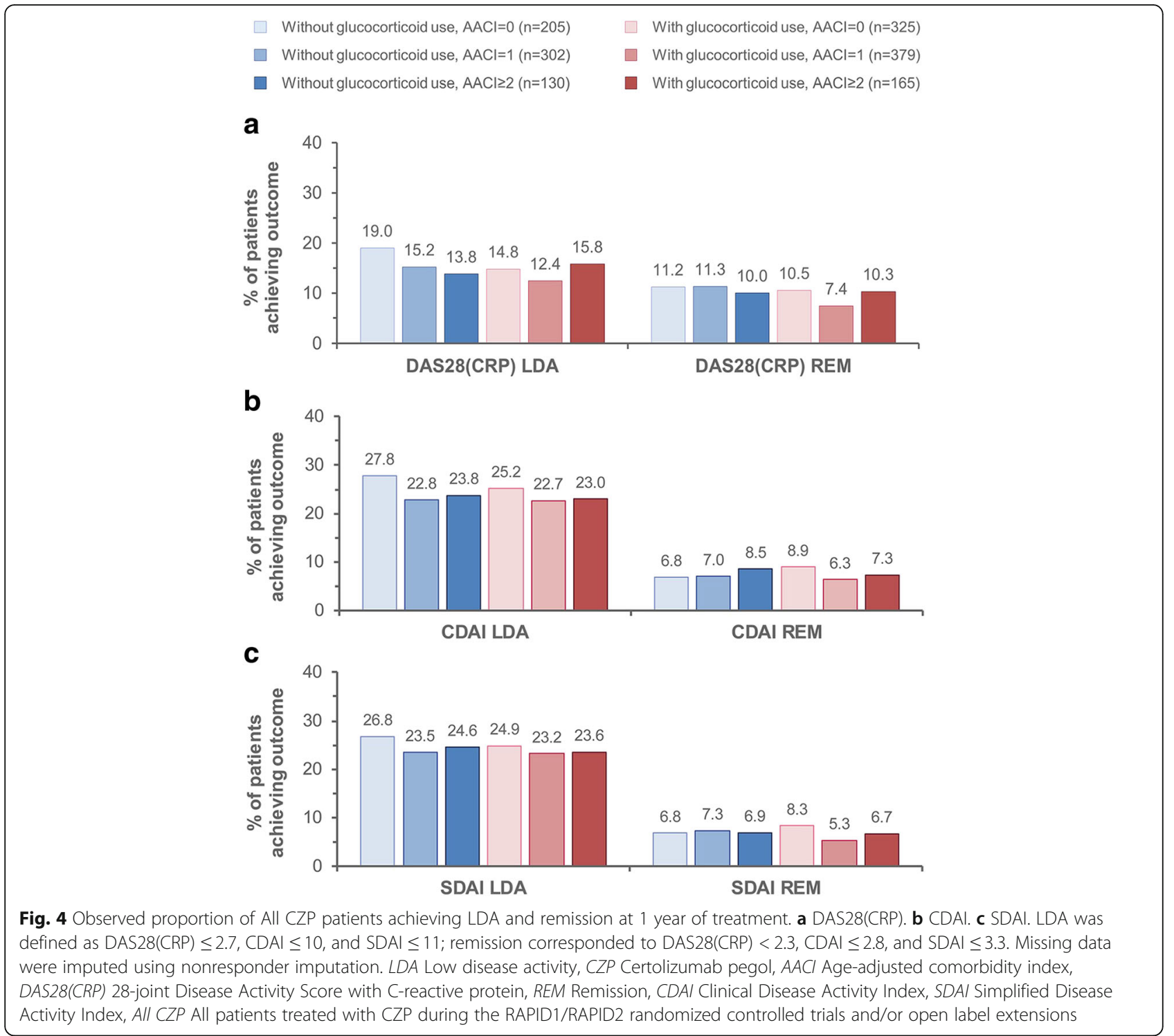

may also have contributed to the underestimation of RABBIT Risk Scores.

At initiation of anti-TNF therapy, prescribers need to consider the infection risk in each individual patient, which must take into account the patient's age, comorbidities, concomitant medications, and clinical history. However, most data currently available consist of mean infection rates observed in relatively large, heterogeneous populations. Although group-level infection rates are informative, they are not always relevant at the individual patient level in clinical practice. This is why stratifying patients according to comorbidity burden and other infection risk factors can help clinicians to make more accurate risk-benefit assessments regarding anti-TNF therapy and ultimately make better-informed treatment decisions at the individual-patient level.
However, in clinical practice, prescribers tend to channel higher-risk patients away from anti-TNF therapy, owing to the commonly held view that older patients or those with more severe comorbidities are less likely to benefit clinically from anti-TNF treatment $[11,39,40]$. However, there is published evidence suggesting that responsiveness to treatment and the incremental infection risk associated with anti-TNFs is similar for higher- and lower-risk groups [41, 42]. Consistent with this, our data showed that the rates of LDA and remission at 1 year of CZP treatment were similar across all AACI risk groups. Although the limited placebo exposure did not allow us to quantify the incremental risk associated with CZP, the results of our study are reassuring for patients with higher AACI scores at baseline and may encourage clinicians to avoid concomitant glucocorticoids to reduce the SIE risk. Further research is needed to examine how the 
disease-modifying effects of CZP treatment, as well as changes in glucocorticoid use, influence the risk of SIEs in a time-dependent manner.

\section{Conclusions}

In this population of patients with moderate to severe active RA and no prior anti-TNF use, the rate of SIEs during CZP treatment was predicted with considerable accuracy using a combination of AACI score and baseline systemic glucocorticoid use, with good discrimination between high- and low-risk patient groups. This SIE risk score may provide a valuable tool for clinicians when considering the risk of infection in individual patients with RA. One of the greatest strengths of our analysis was the use of a large, very comprehensive clinical trial database to derive the AACI. However, we should note that the AACI model has not been validated in a different population. It would be valuable to assess how the AACI performs in other RA trials and in real-world settings.

\section{Additional files}

Additional file 1: Methods: testing of RABBIT Risk Score in the All CZP group. Table S1. Observed number of SIEs in patients categorized by AACl. Figure S1. Predicted and observed SIE rates in All CZP patients according to the RABBIT Risk Score. (PDF $178 \mathrm{~kb}$ )

\section{Abbreviations \\ AACI: Age-adjusted comorbidity index; BMI: Body mass index; CDAl: Clinical Disease Activity Index; Cl: Confidence interval; COPD: Chronic obstructive pulmonary disease; CRP: C-reactive protein; CZP: Certolizumab pegol; DAS28(CRP): 28-joint Disease Activity Score with C-reactive protein; HAQ-DI: Health Assessment Questionnaire Disability Index; HR: Hazard ratio; IR: Incidence rate; LD: Loading dose; LDA: Low disease activity; MedDRA: Medical Dictionary for Regulatory Activities; mTSS: Modified total Sharp score; MTX: Methotrexate; OLE: Open-label extension; PY: Patient-years; RA: Rheumatoid arthritis; RABBIT: Rheumatoid Arthritis Observation of Biologic Therapy (the German biologics register); RAPID: Rheumatoid Arthritis Preventlon of structural Damage; RCT: Randomized controlled trial; SAE: Serious adverse event; SDAI: Simplified Disease Activity Index; SIE: Serious infectious event; TNF: Tumor necrosis factor \\ Acknowledgements \\ The authors thank the patients and their caregivers in addition to the investigators and their teams who contributed to this study. The authors acknowledge Pauline Ralston, MSc, UCB Pharma, Brussels, Belgium, for statistical assistance; and Ricardo Milho, PhD, and Debbie Nixon, DPhil, from Costello Medical, Cambridge, UK, for medical writing and editorial assistance in the preparation of this manuscript for publication based on the authors' input and direction.}

\section{Funding}

This study was funded by UCB Pharma.

\section{Availability of data and materials}

The datasets generated and analyzed for this article were derived from study data available in anonymized format upon reasonable request via the Clinical Study Data Request platform (www.clinicalstudydatarequest.com).

\section{Authors' contributions}

$\mathrm{JRC}, \mathrm{KW}, \mathrm{CO}$ 'B, 'MNN, MdL, and BH contributed to the conception, design, execution, or analysis and interpretation of the data. The authors read and approved the final manuscript to be published after critically revising it for important intellectual content.

Ethics approval and consent to participate

This is a secondary manuscript reporting post hoc analyses of data pooled from the RAPID1 and RAPID2 randomized controlled trials (NCT00152386 and NCT00160602, respectively) and their open-label extensions (NCT00175877 and NCT00160641, respectively). Information regarding ethics approval and patient consent can be found in the corresponding primary publications [20-23]

\section{Competing interests}

JRC has received research grants and consultant fees from Roche, Genentech, UCB Pharma, Janssen, Corrona, Amgen, Pfizer, Bristol-Myers Squibb, Crescendo, and AbbVie. KW has received consultant fees from UCB Pharma, Pfizer, Bristol-Myers Squibb, Eli Lilly, AbbVie, and Roche. CO'B was an employee of UCB Pharma at the time of this work. 'MNN is an employee of UCB Pharma. MdL is an employee of UCB Pharma. BH has received research grants and consultant fees from Abbott, Amgen, Bristol-Myers Squibb, Janssen, Pfizer, Roche, and UCB Pharma. UCB sponsored the study and the development of the manuscript/publication and reviewed the text to ensure that, from UCB's perspective, the data presented in this article are scientifically, technically, and medically supportable; that they do not contain any information that has the potential to damage the intellectual property of UCB; and that the publication complies with applicable laws, regulations, guidelines, and good industry practice.

\section{Publisher's Note}

Springer Nature remains neutral with regard to jurisdictional claims in published maps and institutional affiliations.

\section{Author details}

${ }^{1}$ University of Alabama at Birmingham, Birmingham, AL, USA. ${ }^{2}$ Oregon Health and Science University, Portland, OR, USA. ${ }^{3}$ UCB Pharma, Brussels, Belgium. ${ }^{4}$ Department of Medicine, Centre Hospitalier de l'Université de Montréal, Montréal, QC, Canada.

Received: 24 May 2017 Accepted: 7 November 2017

Published online: 15 December 2017

\section{References}

1. Nam JL, Ramiro S, Gaujoux-Viala C, Takase K, Leon-Garcia M, Emery P, Gossec L, Landewé R, Smolen JS, Buch MH. Efficacy of biological diseasemodifying antirheumatic drugs: a systematic literature review informing the 2013 update of the EULAR recommendations for the management of rheumatoid arthritis. Ann Rheum Dis. 2014;73(3):516-28.

2. Bongartz T, Sutton AJ, Sweeting MJ, Buchan I, Matteson EL, Montori V. Anti-TNF antibody therapy in rheumatoid arthritis and the risk of serious infections and malignancies: systematic review and metaanalysis of rare harmful effects in randomized controlled trials. JAMA. 2006;295(19):2275-85.

3. Leombruno JP, Einarson TR, Keystone EC. The safety of anti-tumour necrosis factor treatments in rheumatoid arthritis: meta and exposure-adjusted pooled analyses of serious adverse events. Ann Rheum Dis. 2009;68(7):1136-45.

4. Singh JA, Cameron C, Noorbaloochi S, Cullis T, Tucker M, Christensen R, Ghogomu ET, Coyle D, Clifford T, Tugwell P, Wells GA. Risk of serious infection in biological treatment of patients with rheumatoid arthritis: a systematic review and meta-analysis. Lancet. 2015;386(9990):258-65.

5. Thompson AE, Rieder SW, Pope JE. Tumor necrosis factor therapy and the risk of serious infection and malignancy in patients with early rheumatoid arthritis: a meta-analysis of randomized controlled trials. Arthritis Rheum. 2011;63(6):1479-85.

6. Greenberg JD, Reed G, Kremer JM, Tindall E, Kavanaugh A, Zheng C, Bishai W, Hochberg MC. Association of methotrexate and tumour necrosis factor antagonists with risk of infectious outcomes including opportunistic infections in the CORRONA registry. Ann Rheum Dis. 2010;69(2):380-6.

7. Listing J, Gerhold K, Zink A. The risk of infections associated with rheumatoid arthritis, with its comorbidity and treatment. Rheumatology. 2013;52(1):53-61.

8. Strangfeld A, Eveslage M, Schneider M, Bergerhausen HJ, Klopsch T, Zink A, Listing J. Treatment benefit or survival of the fittest: what drives 
the time-dependent decrease in serious infection rates under TNF inhibition and what does this imply for the individual patient? Ann Rheum Dis. 2011;70(11):1914-20.

9. Zink A, Askling J, Dixon WG, Klareskog L, Silman AJ, Symmons DP. European biologicals registers: methodology, selected results and perspectives. Ann Rheum Dis. 2009;68(8):1240-6.

10. Cobo-lbáñez T, Descalzo MÁ, Loza-Santamaría E, Carmona L, Muñoz-Fernández S. Serious infections in patients with rheumatoid arthritis and other immunemediated connective tissue diseases exposed to anti-TNF or rituximab: data from the Spanish registry BIOBADASER 2.0. Rheumatol Int. 2014;34(7):953-61.

11. Curtis JR, Xie F, Chen L, Muntner P, Grijalva CG, Spettell C, Fernandes J, McMahan RM, Baddley JW, Saag KG, et al. Use of a disease risk score to compare serious infections associated with anti-tumor necrosis factor therapy among high- versus lower-risk rheumatoid arthritis patients. Arthritis Care Res. 2012:64(10):1480-9.

12. Doran MF, Crowson CS, Pond GR, O'Fallon WM, Gabriel SE. Predictors of infection in rheumatoid arthritis. Arthritis Rheum. 2002;46(9):2294-300.

13. Galloway JB, Hyrich KL, Mercer LK, Dixon WG, Fu B, Ustianowski AP, Watson KD, Lunt M, Symmons DP, Consortium BCC, et al. Anti-TNF therapy is associated with an increased risk of serious infections in patients with rheumatoid arthritis especially in the first 6 months of treatment: updated results from the British Society for Rheumatology Biologics Register with special emphasis on risks in the elderly. Rheumatology. 2011;50(1):124-31.

14. Van Dartel SA, Fransen J, Kievit W, Dutmer EA, Brus HL, Houtman NM, Van De Laar MA, Van Riel PL. Predictors for the 5-year risk of serious infections in patients with rheumatoid arthritis treated with anti-tumour necrosis factor therapy: a cohort study in the Dutch Rheumatoid Arthritis Monitoring (DREAM) registry. Rheumatology. 2013;52(6):1052-7.

15. Haraoui B, Combe B, Champsaur M, Luijtens K, Keystone E. Effects of different steroid doses on adverse events and radiographic progression of certolizumab pegol-treated rheumatoid arthritis patients [abstract]. Ann Rheum Dis. 2013;71 Suppl 3:498.

16. Crowson CS, Hoganson DD, Fitz-Gibbon PD, Matteson EL. Development and validation of a risk score for serious infection in patients with rheumatoid arthritis. Arthritis Rheum. 2012;64(9):2847-55.

17. Zink A, Manger B, Kaufmann J, Eisterhues C, Krause A, Listing J, Strangfeld A. Evaluation of the RABBIT Risk Score for serious infections. Ann Rheum Dis. 2014;73(9):1673-6.

18. Karmiris K, Paintaud G, Noman M, Magdelaine-Beuzelin C, Ferrante M, Degenne D, Claes K, Coopman T, Van Schuerbeek N, Van Assche G, et al. Influence of trough serum levels and immunogenicity on long-term outcome of adalimumab therapy in Crohn's disease. Gastroenterology. 2009;137(5):1628-40.

19. European Medicines Agency (EMA). Annex I: summary of product characteristics (Cimzia). London: EMA; 2013.

20. Keystone E, Heijde D, Mason Jr D, Landewé R, Vollenhoven RV, Combe B, Emery P, Strand V, Mease P, Desai C, et al. Certolizumab pegol plus methotrexate is significantly more effective than placebo plus methotrexate in active rheumatoid arthritis: findings of a fifty-two-week, phase III, multicenter, randomized, double-blind, placebo-controlled, parallel-group study. Arthritis Rheum. 2008;58(11):3319-29.

21. Smolen J, Landewé RB, Mease P, Brzezicki J, Mason D, Luijtens K, van Vollenhoven RF, Kavanaugh A, Schiff M, Burmester GR, et al. Efficacy and safety of certolizumab pegol plus methotrexate in active rheumatoid arthritis: the RAPID 2 study. A randomised controlled trial. Ann Rheum Dis. 2009;68(6):797-804.

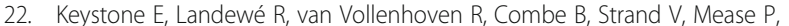
Shaughnessy L, Vanlunen B, van der Heijde D. Long-term safety and efficacy of certolizumab pegol in combination with methotrexate in the treatment of rheumatoid arthritis: 5-year results from the RAPID 1 trial and open-label extension. Ann Rheum Dis. 2014;73(12):2094-100.

23. Smolen JS, van Vollenhoven R, Kavanaugh A, Strand V, Vencovsky J, Schiff M, Landewé R, Haraoui B, Arendt C, Mountian I, et al. Certolizumab pegol plus methotrexate 5-year results from the rheumatoid arthritis prevention of structural damage (RAPID) 2 randomized controlled trial and long-term extension in rheumatoid arthritis patients. Arthritis Res Ther. 2015;17:245.

24. U.S. Food and Drug Administration (FDA). Reporting serious problems to the FDA: what is a serious adverse event? Silver Spring, MD: FDA; 2012. https://www.fda.gov/safety/medwatch/howtoreport/ucm053087.htm. Accessed 15 Feb 2017
25. Bykerk VP, Cush J, Winthrop K, Calabrese L, Lortholary O, de Longueville M, van Vollenhoven $\mathrm{R}$, Mariette $\mathrm{X}$. Update on the safety profile of certolizumab pegol in rheumatoid arthritis: an integrated analysis from clinical trials. Ann Rheum Dis. 2015:74(1):96-103.

26. Charlson ME, Pompei $P$, Ales $K L$, Mackenzie CR. A new method of classifying prognostic comorbidity in longitudinal studies: development and validation. J Chronic Dis. 1987;40(5):373-83.

27. Gönen M, Heller G. Concordance probability and discriminatory power in proportional hazards regression. Biometrika. 2005;92(4):965-70.

28. Doran MF, Crowson CS, Pond GR, O'Fallon WM, Gabriel SE. Frequency of infection in patients with rheumatoid arthritis compared with controls: a population-based study. Arthritis Rheum. 2002;46(9):2287-93.

29. Quinn MA, Conaghan PG, O'Connor PJ, Karim Z, Greenstein A, Brown A, Brown C, Fraser A, Jarret S, Emery P. Very early treatment with infliximab in addition to methotrexate in early, poor-prognosis rheumatoid arthritis reduces magnetic resonance imaging evidence of synovitis and damage, with sustained benefit after infliximab withdrawal: results from a twelvemonth randomized, double-blind, placebo-controlled trial. Arthritis Rheum. 2005;52(1):27-35.

30. Jayakumar K, Norton S, Dixey J, James D, Gough A, Williams P, Prouse P, Young A. Sustained clinical remission in rheumatoid arthritis: prevalence and prognostic factors in an inception cohort of patients treated with conventional DMARDs. Rheumatology. 2012;51(1):169-75.

31. Rogers MA, Greene MT, Young VB, Saint S, Langa KM, Kao JY, Aronoff DM. Depression, antidepressant medications, and risk of Clostridium difficile infection. BMC Med. 2013;11:121.

32. Dixon WG, Abrahamowicz M, Beauchamp ME, Ray DW, Bernatsky S, Suissa S, Sylvestre MP. Immediate and delayed impact of oral glucocorticoid therapy on risk of serious infection in older patients with rheumatoid arthritis: a nested case-control analysis. Ann Rheum Dis. 2012;71(7):1128-33.

33. Singh JA, Saag KG, Bridges Jr SL, Akl EA, Bannuru RR, Sullivan MC, Vaysbrot E, McNaughton C, Osani M, Shmerling RH, et al. 2015 American College of Rheumatology Guideline for the Treatment of Rheumatoid Arthritis. Arthritis Rheumatol. 2016;68(1):1-26.

34. Au K, Reed G, Curtis JR, Kremer JM, Greenberg JD, Strand V, Furst DE. High disease activity is associated with an increased risk of infection in patients with rheumatoid arthritis. Ann Rheum Dis. 2011;70(5):785-91.

35. Emery P, Gallo G, Boyd H, Morgan CL, Currie CJ, Poole CD, Nab HW. Association between disease activity and risk of serious infections in subjects with rheumatoid arthritis treated with etanercept or diseasemodifying anti-rheumatic drugs. Clin Exp Rheumatol. 2014;32(5):653-60.

36. Askling J, Fored CM, Brandt L, Baecklund E, Bertilsson L, Feltelius N, Coster L, Geborek P, Jacobsson LT, Lindblad S, et al. Time-dependent increase in risk of hospitalisation with infection among Swedish RA patients treated with TNF antagonists. Ann Rheum Dis. 2007;66(10):1339-44.

37. Curtis JR, Patkar N, Xie A, Martin C, Allison JJ, Saag M, Shatin D, Saag KG. Risk of serious bacterial infections among rheumatoid arthritis patients exposed to tumor necrosis factor a antagonists. Arthritis Rheum. 2007:56(4):1125-33.

38. Klareskog L, Gaubitz M, Rodriguez-Valverde V, Malaise M, Dougados M, Wajdula J. Assessment of long-term safety and efficacy of etanercept in a 5-year extension study in patients with rheumatoid arthritis. Clin Exp Rheumatol. 2011;29(2):238-47.

39. Radner H, Yoshida K, Hmamouchi I, Dougados M, Smolen JS, Solomon DH. Treatment patterns of multimorbid patients with rheumatoid arthritis: results from an international cross-sectional study. J Rheumatol. 2015;42(7): 1099-104.

40. Turesson C. Comorbidity in rheumatoid arthritis. Swiss Med Wkly. 2016;146: w14290.

41. Bathon JM, Fleischmann RM, Van der Heijde D, Tesser JR, Peloso PM, Chon $Y$, White B. Safety and efficacy of etanercept treatment in elderly subjects with rheumatoid arthritis. J Rheumatol. 2006:33(2):234-43.

42. Koller MD, Aletaha D, Funovits J, Pangan A, Baker D, Smolen JS. Response of elderly patients with rheumatoid arthritis to methotrexate or TNF inhibitors compared with younger patients. Rheumatology. 2009;48(12):1575-80. 